\title{
Integração Espacial no Mercado Brasileiro de Soja em Grão, no Período 1994-2008
}

\section{Spatial Integration in Brazilian Soybean Market in the Period 1994-2008}

\author{
Talles Girardi de Mendonça* \\ João Eustáquio de Lima** \\ Viviani Silva Lírio** \\ Vanessa da Fonseca Pereira"****
}

Resumo: As alterações ocorridas no mercado agrícola brasileiro nas décadas de 1980 e 1990, referentes à menor intervenção governamental e à liberalização comercial, modificaram o seu processo de formação de preços. Neste estudo, buscou-se verificar o processo de formação de preços praticados nos principais estados produtores de soja em grão no Brasil, que são, em ordem de importância, Mato Grosso, Paraná, Rio Grande do Sul, Goiás, Mato Grosso do Sul, Minas Gerais, São Paulo e Santa Catarina. Para isso, procedeu-se ao teste de cointegração e à estimação de um Modelo de Correção de Erro Vetorial (VEC). Os preços utilizados foram obtidos na Fundação Getúlio Vargas (FGV) e referem-se ao período de agosto de 1994 a agosto de 2008. Os resultados indicaram que o mercado brasileiro de soja em grão é integrado, porém de forma imperfeita. Foi constatada interdependência na formação de preços da maior parte dos estados considerados, sendo Santa Catarina, São Paulo, Minas Gerais e Paraná os que apresentaram maior grau de interdependência.

Palavras-chave: Integração no mercado de soja. Formação de preços. Brasil.

Abstract: The changes in the Brazilian agricultural market in the 1980s and 1990s, regarding minor government intervention and trade liberalization, have changed its pricing process. In this study, we aimed to verify the pricing process of the main soybean producing states in Brazil, which are, in order of importance, Mato Grosso, Paraná, Rio Grande do Sul, Goiás, Mato Grosso do Sul, Minas Gerais, São Paulo and Santa Catarina. To do so, we performed a cointegration

\footnotetext{
* Doutorando em Economia Aplicada pela Universidade Federal de Viçosa (UFV). E-mail: tallesgm@yahoo.com.br

** Professor Titular do Departamento de Economia Rural da UFV. E-mail: jelima@ufv.br

*** Professor Adjunto do Departamento de Economia Rural da UFV. E-mail: vslirio@ufv.br

**** Doutoranda em Economia Aplicada pela UFV. E-mail: vanessajf@acessa.com
} 
test and the estimation of a Vector Error Correction model (VEC). Prices were obtained from Fundação Getúlio Vargas (FGV) and refer to the period from August 1994 to August 2008. The results indicated that the Brazilian soybean market is integrated, however imperfectly. Interdependence was found in the pricing process of most states considered, and Santa Catarina, São Paulo, Minas Gerais and Paraná had the highest degree of interdependence.

Keywords: Soybean market integration. Pricing formation process. Brazil.

JEL Classification: L11; Q10; Q13.

\section{1 lntrodução}

A produção agrícola, aliada às atividades de comercialização, armazenamento e processamento, desempenha papel de grande relevância na economia nacional. A vantagem comparativa que o país possui na agricultura permitiu ao Brasil ocupar posição de destaque no cenário internacional, como exportador de alimentos e de matérias-primas e derivados.

A partir da década de 1980, quando vários mercados agrícolas passaram a sofrer menor intervenção governamental, a dinâmica de formação de preços nesses mercados foi afetada. Além disso, a liberalização comercial os tornou mais suscetíveis a variações de preços no mercado internacional.

Nesse contexto, a elaboração de estudos que envolvem a dinâmica e a interdependência de preços agrícolas tornou-se relevante e expressiva. Vários trabalhos foram realizados no intuito de verificar a integração entre o mercado nacional e internacional de determinados produtos agrícolas, bem como a integração de mercados regionais que permitem verificar o processo de transmissão de preços entre os mercados considerados. No primeiro grupo de trabalhos, podem ser citados Margarido, Turolla e Fernandes (2001), que estudaram a soja em grão brasileira e norte-americana; Coelho (2004), que pesquisou o algodão brasileiro, utilizando séries de preços no atacado e índices representativos do preço desse produto no mercado externo; e Cunha (2008), que analisou a integração no mercado internacional de café arábica e robusta, utilizando preços ao produtor dos principais países produtores e duas proxies dos preços internacionais para os dois tipos de café analisados, entre outros. No segundo grupo, podem ser citados Pereira (2005), que analisou a integração espacial no mercado brasileiro de boi gordo, considerando os principais estados produtores; Nogueira et al. (2005), que verificaram a integração dos dois principais mercados produtores de café arábica, Minas Gerais e São Paulo; e Rosado (2006), 
que realizou análise de integração semelhante para os principais estados produtores de suínos.

Faminow e Benson (1990) definiram mercados integrados como aqueles em que os preços são determinados de maneira interdependente, ou seja, as alterações de preços em um mercado são transmitidas aos preços de outros. De forma análoga, segundo Fackler e Goodwin (2001) e González-Rivera e Helfand (2001), localidades espacialmente separadas, que comercializam o mesmo produto, formarão um mercado integrado, se compartilharem informações, de longo prazo, semelhantes. Assim, seus preços estarão em função da oferta e demanda local e das demais localidades que compõem o mesmo mercado.

A análise de integração de mercados, conforme definida anteriormente, tem importantes implicações na geração de informações relevantes para os participantes dos mercados agrícolas e também para o governo. Santana (1998) enfatizou que o conhecimento do grau de integração de mercado pode auxiliar no delineamento de políticas agrícolas gerais e específicas. De Zen (1997) destacou que a dinamicidade dos mercados regionais e a integração destes favorecem o desenvolvimento de modernas técnicas de comercialização, especialmente nos mercados futuros. Goodwin e Schroeder (1991) ressaltaram a importância da análise de integração para verificação da eficiência dos mercados. Segundo esses autores, mercados que não são integrados podem carregar informações imprecisas de preços, que podem distorcer as decisões de comercialização dos produtores e contribuir para movimentação ineficiente de produtos. González-Rivera e Helfand (2001) destacaram que a elevação do grau de integração pode aumentar a renda dos produtores, por permitir especialização crescente e comércio, além de aumentar o bem-estar dos consumidores, pois reduz a variabilidade de preço dos bens. Nogueira et al. (2005) ressaltaram a importância de verificar a integração para a tomada de decisão de comercialização dos agentes de mercado, sendo essa relevância tanto maior quanto mais importante for o mercado estudado para a economia nacional.

Desse modo, a análise de integração das regiões produtoras de soja em grão no País é de grande relevância, na medida em que este segmento do setor agrícola desempenha papel importante no processo de desenvolvimento econômico nacional. Além disso, na maior parte dos estudos de integração, realizados para soja, constatou-se a questão da interdependência do mercado nacional e internacional.

O Brasil, ao lado dos Estados Unidos e da Argentina, é um dos principais produtores e exportadores de soja em grão. Segundo dados do AGRIANUAL (2010), referentes à safra 2008/09, os Estados Unidos produziram 80,5 milhões de toneladas, ao passo que Brasil e Argentina 
produziram 57 e 32 milhões, respectivamente. As exportações também concentram-se nesses países, tendo os Estados Unidos exportado 34,4 milhões de toneladas; o Brasil, 27,8 milhões; e a Argentina, 6 milhões, na safra 2008/09. O valor, em dólares, das exportações brasileiras de soja em grão atingiu US $\$ 11,4$ bilhões, e seus principais derivados, farelo e óleo, US\$ 4,5 bilhões e US\$ 1,04 bilhão, respectivamente (BRASIL, 2009). Esses dados evidenciam a dinamicidade das cadeias produtivas responsáveis por esses produtos.

A soja desempenha papel importante no desenvolvimento regional do País, já que sua produção se distribui por todo o território nacional, sendo os principais produtores, em ordem de importância, Mato Grosso, Paraná, Rio Grande do Sul, Goiás e Mato Grosso do Sul. De acordo com dados da CONAB (2009), esses estados produziram, na safra 2008/2009, $17,9,9,5,7,9,6,8$ e 4,1 milhões de toneladas, respectivamente.

Dadas essas considerações, o objetivo do presente trabalho foi analisar a integração espacial do mercado brasileiro de soja. Especificamente, pretendeu-se verificar se os mercados regionais são espacialmente integrados e estudar a dinâmica da transmissão de preços entre eles. Vale ressaltar que, além das regiões citadas, foram considerados os estados de Minas Gerais, São Paulo e Santa Catarina. Para escolha dos estados a serem incluídos na análise, observou-se a disponibilidade de dados nas fontes consultadas, além da importância relativa de cada estado na produção nacional.

$\mathrm{Na}$ operacionalização da análise, foram utilizados preços recebidos pelos produtores de soja em grão, obtidos da Fundação Getúlio Vargas (FGVDADOS, 2008) e referentes ao período de agosto de 1994 a agosto de 2008. Essas séries foram logaritmizadas para obter, diretamente, as elasticidades de transmissão de preços entre os mercados. O período selecionado levou em conta a disponibilidade de dados e a estabilização da economia, iniciada em julho de 1994.

Além desta introdução, o trabalho está dividido em quatro outras seções. Nas próximas seções, 2 e 3, estão os principais aspectos teóricos e metodológicos que deram suporte ao trabalho. Na quarta seção, são apresentados e discutidos os principais resultados obtidos, e, na quinta, algumas conclusões.

\section{Referencial Teórico}

Segundo Nogueira et al. (2005), a origem da ideia de integração de mercados está na Lei do Preço Único (LPU). Em linhas gerais, essa lei postula que dois bens, idênticos, vendidos em países diferentes, e na ausência de barreiras ao comércio e de custos de transporte, devem 
ser vendidos ao mesmo preço, quando seus preços forem expressos na mesma moeda (KRUGMAN; OBSTFELD, 2005). Ao colocar esse conceito, os autores claramente referiram-se a produtos comercializados no mercado internacional. Entretanto, o conceito também aplica-se a bens comercializados entre regiões de um mesmo país, bastando que ocorra o processo de arbitragem.

Esse processo consiste em adquirir uma mercadoria em um local onde ela for mais barata e vendê-la onde ela for mais cara, após compensar os custos de transferência.

O processo assegurará que os preços de um bem homogêneo, em quaisquer das duas localidades, diferirão, no máximo, em relação ao custo de se mudar o bem de uma região com menor preço para outra com maior preço. O conceito pode ser formalizado da seguinte forma:

$$
P_{j}-P_{i} \leq r_{i j}
$$

em que $P_{j}$ é o preço do bem no local j; $P_{i}$ é o preço do bem no local $i$; e $r_{i j}$ são os custos de se transportar o bem da localidade $i$ para a $j$. Essa condição é denominada condição de arbitragem espacial e constitui um conceito de equilíbrio (KRUGMAN; OBSTFELD, 2005).

Existem diferentes versões para a LPU. A primeira está relacionada com a LPU fraca, que ocorre quando se verifica a condição de arbitragem espacial $\left(P_{j}-P_{i} \leq r_{i j}\right)$; a segunda é a LPU forte, em que se presume que o comércio seja contínuo e a condição de arbitragem seja garantida com regularidade, isto é, mantém-se como uma igualdade $\left(P_{\mathrm{j}}-P_{\mathrm{i}}=r_{\mathrm{ij}}\right)$. A terceira versão é a LPU agregada, enunciada em termos de índices de preços, sendo conhecida como Paridade do Poder de Compra (PPC) (FACKLER; GOODWIN, 2001).

Em geral, a LPU forte é a mais testada, já que constitui um teste de integração perfeita. Essa forma de integração ocorre quando a transmissão de preços entre as localidades é igual a 1, ou seja, toda mudança de preço em uma localidade é transmitida, integralmente, às demais.

Vale ressaltar que, apesar de ser utilizada em muitos trabalhos empíricos como suporte teórico, a LPU apresenta alguns problemas. De acordo com Ravallion (1986, apud CUNHA, 2008), o processo de arbitragem dificilmente seria eficiente, em razão de barreiras comerciais, informação imperfeita e aversão ao risco. Além disso, o autor destaca que a maioria das firmas possui algum poder de mercado, já que em grande parte dos mercados a pressuposição de competição perfeita não se verifica.

Em razão disso, Fackler e Goodwin (2001) ressaltaram que a integração do mercado deve ser entendida como uma medida de grau, 
em que choques de demanda e de oferta que surgem em uma região são transmitidos para outra. Os autores destacaram que se os choques em determinada região forem integralmente transmitidos a outros mercados tem-se a integração perfeita, do contrário, pode-se concluir apenas sobre a interdependência dos preços. Desse modo, a integração de mercados refere-se à interdependência de preços entre localidades espacialmente separadas, ou seja, os preços de um mercado integrado sofrerão influências não apenas das condições de oferta e demanda locais, mas também das condições das demais localidades.

O conceito proposto pelos autores foi formalizado pela razão de transmissão de preços, $\mathrm{R}_{\mathrm{AB}}$, associada a um choque hipotético na região $\mathrm{A}, \varepsilon_{A}$, que poderia representar um choque do lado da oferta ou da demanda. Sua expressão é dada por (2):

$$
R_{A B}=\frac{\frac{\partial P_{B}}{\partial \varepsilon_{A}}}{\frac{\partial P_{A}}{\partial \varepsilon_{A}}}=\frac{\partial P_{B}}{\partial P_{A}},
$$

em que $\frac{\partial P_{B}}{\partial \varepsilon_{A}}$ é a variação infinitesimal do choque econômico que modifica o preço do bem na região " $\mathrm{B}$ "; $\frac{\partial P_{A}}{\partial \varepsilon_{A}}$ é a variação infinitesimal do choque econômico que modifica o preço do bem na região "A"; e $R_{A B}$ é a razão de transmissão associada ao choque considerado. Se $R_{A B}$ for igual à unidade, uma mudança do preço de "A" será transmitida, na mesma intensidade, a "B", e as duas localidades serão perfeitamente integradas.

Desse modo, a movimentação conjunta de preços ou, de forma análoga, sua interdependência é condição para a integração, o que indica que ela não depende, exclusivamente, da arbitragem ou de que os mercados estejam ligados diretamente por comércio.

De fato, a integração espacial de mercados pode também ocorrer por meio da transmissão indireta de preços, ou seja, não é necessário que duas regiões sejam parceiras diretas de comércio para que exista alto grau de integração entre elas. Essas duas localidades podem estar ligadas pelo processo de comercialização de ambas com uma terceira região, o que seria suficiente para uma transmissão de preços indireta entre elas, já que todas, nesse caso, fariam parte de uma mesma rede de comércio e de informações (FACKLER; GOODWIN, 2001).

González-Rivera e Helfand (2001) destacaram que um mercado com $K$ localidades, geograficamente distintas, será considerado inte- 
grado se existir um fluxo físico de bens, direta ou indiretamente, que conecte as K regiões; e se as localidades possuírem um vetor de preços, $P_{t}=\left\{p_{1 t}, p_{2 t}, \ldots, p_{k t}\right\}$, que possa ser decomposto em um componente permanente, ou de longo prazo, e um componente transitório, ou de curto prazo. Segundo os autores, dado que $P_{t}$ é I(1), a decomposição do vetor de preços resulta em:

$$
P_{t}=A_{k x s} f_{t}+\tilde{P}_{t}
$$

em que $f_{t}$ é um vetor $s \times 1$ de fatores de raízes unitárias comuns $s(s<k)$; e $\tilde{P}_{t}$ é um vetor (kx1) de componentes estacionários, ou seja, $\mathrm{I}(0)$.

Cada elemento no vetor $P_{t}$ pode ser explicado por uma combinação linear de um número menor de fatores comuns $f_{j t}$ (componentes permanentes) I(1) com um componente transitório I(0), por exemplo, $p_{i t}=\sum_{j=1}^{s} a_{i j} f_{j t}+\tilde{p}_{i t}$. No longo prazo, tem-se que as variáveis $p_{i t}$ movemse conjuntamente, uma vez que compartilham a mesma tendência estocástica. Se for esse o caso, podem-se definir as $K$ localidades consideradas como parte de um mesmo mercado econômico integrado. As implicações dessas condições na análise proposta neste trabalho, com base na análise de cointegração, serão delineadas no item subsequente.

\section{Modelo Analítico}

Segundo Nogueira et al. (2005), embora a não observação da LPU, no curto prazo, tenha conotação de ineficiência, graças à lentidão da ação da arbitragem, essa parece ser a regra, e não a exceção. Isso porque o processo de arbitragem leva algum tempo para se efetivar.

Portanto, a análise de cointegração apresenta a vantagem de mostrar a integração de mercados no longo prazo. Além disso, permite a análise baseada em séries não estacionárias.

Conforme ressaltado por Enders (1995), a ideia que antecede o conceito de cointegração é que variáveis não estacionárias podem caminhar juntas, ou seja, apresentar trajetórias temporais interligadas, de modo que, no longo prazo, tenham relação de equilíbrio. Dessa forma, a existência de cointegração é um indício de interdependência e, portanto, de integração dos preços em um mercado. Assim, a análise realizada neste trabalho recaiu sobre a análise de cointegração. 
Para operacionalização da análise, o primeiro passo é a definição da ordem de integração das séries, pois para que a análise de cointegração seja realizada é necessário que as séries possuam a mesma ordem de integração.

A determinação da ordem de integração para as séries de preços logaritmizadas foi implementada por meio do teste Dickey-Fuller Aumentado (ADF), ${ }^{1}$ proposto por Dickey e Fuller (1981).

Definida a ordem de integração das séries de preços, pode-se realizar o teste de cointegração, desde que, conforme ressaltado, as ordens de integração sejam as mesmas. Neste trabalho, optou-se pelo teste de cointegração de Johansen (1988), uma vez que pretendeu-se verificar a interdependência dos preços por meio de um modelo multivariado.

O teste é conduzido por meio da estimação de um modelo de Correção de Erro Vetorial (VEC), que é uma versão reparametrizada de um modelo de Autorregressão Vetorial (VAR).

Enders (1995) destacou que no modelo VAR tem-se uma equação para cada variável (no caso deste estudo, as séries de preços em cada estado), em função de seus valores passados e dos valores defasados das outras variáveis. Assim, em sua representação matricial, um modelo VAR, com $k$ variáveis e $p$ defasagens, pode ser escrito da seguinte forma:

$$
P_{t}=\alpha+\theta_{1} P_{t-1}+\theta_{2} P_{t-2}+\ldots+\theta_{p} P_{t-p}+\varepsilon_{t}
$$

em que $P_{t}$ é um vetor $(k \times 1)$ de variáveis endógenas; $P_{t-j}, j=1,2, \ldots, p$ são vetores ( $k \times 1)$ de variáveis defasadas; $\alpha$ é um vetor $(k \times 1)$ de interceptos; $\theta_{j}, j=1,2, \ldots, p$ são matrizes $(k x k)$ de coeficientes a serem estimados; e $\varepsilon_{\mathrm{t}}$ é um vetor $(k \times 1)$ de erros aleatórios.

A versão reparametrizada do modelo VAR, conforme definido em (4), é dada por

$$
\Delta P_{t}=\mu+\Pi P_{t-1}+\Gamma_{1} \Delta P_{t-1}+\ldots+\Gamma_{p-1} \Delta P_{t-(p-1)}+\varepsilon_{t}
$$

em que $\Gamma_{\mathrm{i}}, i=1,2, \ldots, p-1$ e $\Pi$ são matrizes $(k x k)$, sendo $\Gamma_{i}=-\sum_{j=i+1}^{p} \theta_{j} \mathrm{e}$ $\Pi=-\left(I_{k}-\sum_{i=1}^{p} \theta_{i}\right)$.

O método de Johansen (1988) consiste em determinar o posto da matriz $\Pi$ ou o número de raízes características $(\lambda)$ diferentes de zero. Segundo esse procedimento, para as variáveis serem cointegradas as linhas de $\Pi$ não podem ser todas linearmente independentes; elas têm de ser dependentes, porque não se pode ter $k$ combinações lineares

A descrição completa do teste pode ser obtida em Enders (1995). 
(relações de cointegração) para $k$ variáveis cointegradas. Desse modo, $\Pi$ deve ser singular, ou seja, seu determinante deve ser nulo e, nesse caso, o posto ou rank (r) da matriz $\Pi$ é menor que o número de variáveis $k$.

Para que haja integração entre os mercados estudados, é necessário não apenas cointegração entre os preços $(r<k)$, mas também $(k-1)$ vetores de cointegração. Se tal condição for satisfeita, os preços nos diferentes mercados compartilharão a mesma informação de longo prazo, o que é um requisito para a existência de integração entre eles e, portanto, para a existência de um mercado integrado.

A necessidade de que existam $k$ - 1 vetores de cointegração reside no fato de que a relação (3), apresentada no referencial teórico e conhecida como representação do fator comum, só se verifica se existirem $k-s$ vetores de cointegração entre os elementos do vetor $P_{t}$. Assim, se existirem $k$ séries de preços, poderão existir $r$ vetores de cointegração e $s$ fatores de raízes unitárias comuns (tendências comuns), de modo que $r+s=k$ ou $r=k-s$.

González-Rivera e Helfand (2001) destacaram que a correta definição do mercado econômico integrado requer a existência de uma única tendência estocástica comum a todos os preços. Deste modo, para que determinado mercado seja integrado, é necessário que todos os seus preços compartilhem a mesma tendência de longo prazo, ou seja, $s=1$.

Antes de prosseguir, é necessário ressaltar que, como não se conhece a priori a interdependência dos preços praticados nos mercados considerados e, portanto, a extensão do mercado brasileiro de soja em grão, a abordagem multivariada, adotada neste estudo, é mais apropriada que a abordagem bivariada baseada na estimação de regressões por pares de preços. O enfoque multivariado permite determinar, de maneira adequada, se os preços praticados em diferentes localidades compartilham a mesma tendência estocástica. Esse aspecto foi ressaltado por González-Rivera e Helfand (2001).

Se houver cointegração entre os preços, a matriz $\Pi$ poderá ser reescrita da seguinte forma:

$$
\Pi_{k x k}=\alpha_{k x r} \beta_{r x k}^{\prime}
$$

em que $\alpha$ é uma matriz de coeficientes de ajustamento para o equilíbrio de longo prazo; e $\beta$ é uma matriz de vetores de cointegração.

Substituindo (6) em (5), tem-se um Modelo de Correção de Erro Vetorial (VEC) com a seguinte forma:

$$
\Delta P_{t}=\mu+\alpha \beta^{\prime} P_{t-1}+\Gamma_{1} \Delta P_{t-1}+\ldots+\Gamma_{p-1} \Delta P_{t-(p-1)}+\varepsilon_{t}
$$


em que $\beta^{\prime} P_{t-1}$ corresponde às $r$ relações de cointegração; e $\Gamma_{\mathrm{i}}$ são matrizes de coeficientes que definem a dinâmica de curto prazo entre os preços.

A determinação do número de raízes características $(\lambda)$ diferentes de zero na matriz $\Pi$, que corresponde ao número de vetores de cointegração (r), é obtida por meio de dois testes, o teste do traço e o teste do máximo autovalor, equações (8) e (9), ambos propostos por Johansen (1988).

$$
\begin{aligned}
& \lambda_{\text {trace }}=-T \sum_{i=r+1}^{k} \ln \left(1-\hat{\lambda}_{i}\right) \\
& \lambda_{\max }=-T \ln \left(1-\hat{\lambda}_{r_{0}+1}\right)
\end{aligned}
$$

em que $T$ é o número de observações usadas no ajustamento; e $\lambda_{i}$ é a i-ésima raiz característica de $\Pi$. O teste do traço $\left(\lambda_{\text {trace }}\right)$ verifica a hipótese de que existem, no máximo, $r$ vetores de cointegração $\left(H_{0}: r \leq r_{0}\right.$, contra $\left.H_{\alpha}: r>r_{0}\right)$. O teste de máximo autovalor $\left(\lambda_{\max }\right)$, por sua vez, testa a hipótese nula de que há $r$ vetores de cointegração, contra a hipótese alternativa de que há $(r+1)$.

Encontrado o número de relações de cointegração, conforme requer a teoria da integração de mercados $(r=k-1)$, procedeu-se à estimação do modelo VEC, no intuito de determinar a dinâmica da transmissão de preços ou o padrão de interdependência dos preços nos mercados analisados.

O modelo VEC, cuja especificação é dada pela equação (7), fornece informações a respeito dos ajustamentos em $P_{t}$ no curto e no longo prazo, por meio das estimativas das matrizes de coeficientes $\Gamma_{i} \mathrm{e}$ $\Pi=\alpha \beta^{\prime}$, respectivamente.

As matrizes $\Gamma_{i}$ contêm as elasticidades de ajustamento no curto prazo e, portanto, representam o efeito, de curto prazo, de variações de preço entre os mercados. Vale ressaltar que boa parte desses coeficientes, em geral, não é significativa, já que, conforme destacado anteriormente, os ajustes de preços entre mercados levam algum tempo para se efetivarem. A matriz $\beta$, chamada matriz de cointegração, mostra as relações que devem ser asseguradas entre as variáveis no equilíbrio de longo prazo. Assim, os elementos de $\beta$ determinam como cada variável cointegrada faz o sistema convergir, após um choque, em direção ao equilíbrio no longo prazo. Já a matriz $\alpha$, fornece informações sobre a velocidade do ajustamento dos preços em determinada localidade $i$, 
dado um desequilíbrio nas demais, sendo conhecida como matriz de coeficientes de ajustamento.

Nesse ponto, é importante destacar que quando cada localidade responde aos desequilíbrios de todas as outras há grande interdependência dos preços. Nesse caso, todos os coeficientes da matriz $\alpha$ serão significativos. Entretanto, quando determinada localidade atua na formação de preços, influenciando as demais, sem ser influenciada por elas, os coeficientes de ajustamento, referentes aos preços praticados nessa localidade, serão não significativos.

Desse modo, a análise baseada na estimação do modelo VEC requer a realização de testes de significância sobre os parâmetros $\alpha \mathrm{e}$ $\beta$, para cada uma das equações de preços que compõem o sistema. A estatística do teste é a razão de verossimilhança (LR):

$$
L R=2\left[\ln (L)-\ln \left(L^{*}\right)\right] \sim \chi_{m}^{2}
$$

em que $\ln (L)$ refere-se ao logaritmo natural do valor da função de verossimilhança irrestrita; $\ln \left(L^{*}\right)$, ao logaritmo natural da função de verossimilhança, que obedece às restrições impostas pela hipótese nula; e $\chi^{2}{ }_{m}$, aos valores críticos da distribuição de quiquadrado com $m$ graus de liberdade (número de restrições impostas pela hipótese nula). Os valores calculados de $L R$ devem ser comparados aos valores críticos $\chi^{2}{ }_{m}, \mathrm{e}$ se forem menores a hipótese nula de que os parâmetros estimados são estatisticamente iguais a zero não será rejeitada.

O teste também pode ser utilizado para testar a hipótese de integração perfeita; nesse caso, a hipótese nula coloca que a variação de preços, ocorrida em determinado mercado, é integralmente repassada aos demais.

\section{Resultados e Discussões}

Esta seção destina-se à apresentação e análise dos principais resultados obtidos. Inicialmente, apresentam-se a análise gráfica das séries utilizadas, o teste de cointegração e os testes de restrições sobre os parâmetros $\alpha \mathrm{e} \beta$, realizados para verificar a questão da integração no mercado brasileiro de soja em grão. Em seguida, apresentam-se as estimativas dos vetores de cointegração, dos coeficientes de ajustamento e os testes de integração perfeita, para analisar a dinâmica da transmissão de preços. 


\subsection{Verificação da Integração no Mercado Brasileiro de Soja em Grão}

Conforme relatado anteriormente, a existência de integração de mercados requer a existência de uma tendência comum, ou seja, comportamento semelhante entre as séries de preços ao longo do tempo. Esse fato torna a análise gráfica das séries de preços um complemento à análise econométrica formal.

A Figura 1 apresenta a evolução dos preços no período analisado e fornece um indicativo de que os preços aos produtores de soja em grão, nos principais estados considerados na análise, apresentam um comportamento comum ao longo do tempo, primeiro indício da integração desses mercados.

Entretanto, conforme destacado, a análise gráfica é um procedimento complementar, sendo necessários procedimentos econométricos para obtenção de conclusões a respeito da integração.

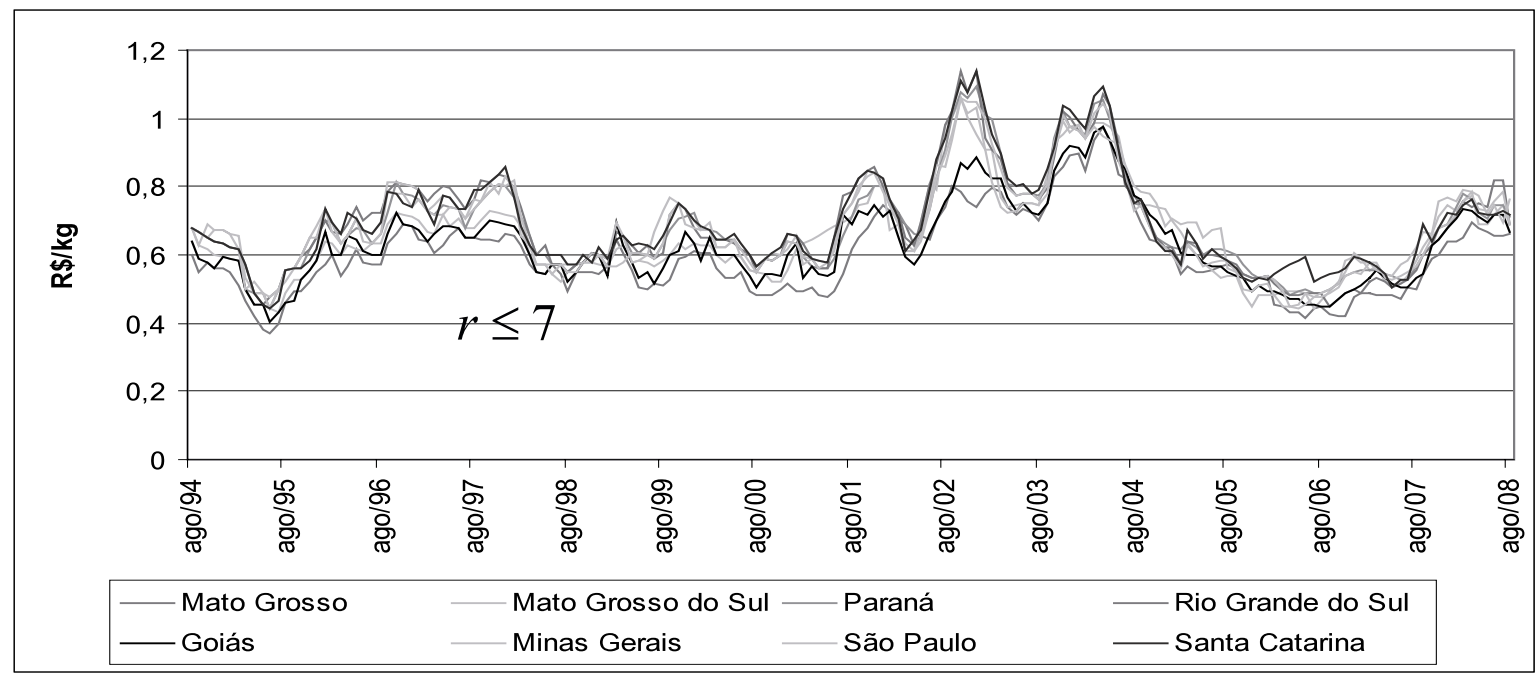

Figura 1 - Preços de soja em grão recebidos pelos produtores, no período de agosto de 1994 a agosto de 2008 , em $\mathrm{R} \$$ por quilo, a preços de agosto de 2008 , nos Estados considerados na análise

Fonte: Elaborado pelos autores com base nos dados da FGV (2008).

O procedimento inicial em análises de integração consiste na verificação da ordem de integração das séries de preços utilizadas. Neste trabalho, tal procedimento foi realizado com base no teste ADF, considerando-se, para a especificação da equação do teste, a presença de intercepto e a ausência de tendência. Além disso, considerou-se um nível de significância de 1\%. Essa decisão baseou-se em Enders (1995), que destacou que a escolha de um nível de significância elevado torna o teste mais potente. A mesma opção foi feita em outros estudos, 
em função da potência do teste, podendo-se destacar os trabalhos de González-Rivera e Helfand (2001), Nogueira et al. (2005), Cunha (2008) e Mattos (2008).

As séries analisadas foram não estacionárias em nível e estacionárias em primeira diferença, considerando-se um nível de significância de 1\% (Tabela 1). Esses resultados permitem concluir que todas as séries apresentam a mesma ordem de integração, ou seja, I(1), que é um requisito para a realização de análises de cointegração.

Verificada a ordem de integração das séries, procedeu-se à análise de cointegração, por meio do procedimento de Johansen (1988), partindo-se do modelo VAR reparametrizado, com o objetivo de identificar se os preços recebidos pelos produtores, nos estados selecionados, apresentam a mesma tendência de comportamento e se, portanto, fazem parte do mesmo mercado econômico, ou seja, do mercado brasileiro de soja em grão. Na escolha das defasagens para o modelo, os três critérios utilizados, Akaike, Schwartz e Hannan-Quinn, indicaram que apenas uma defasagem deveria ser incluída no modelo $\mathrm{VAR}$, o que corresponderia à estimação de um modelo VEC, de ordem . Entretanto, este modelo apresentou problema após o teste de autocorrelação (Multiplicador de Lagrange de Breusch-Godfrey) e, assim, procedeu-se à estimação de um VEC, de ordem 1 , que corrigiu o problema.

Tabela 1 - Teste ADF para o Logaritmo das Séries de Preço de Soja em Grão

\begin{tabular}{lllll}
\hline \multirow{2}{*}{ Estados } & Nivel & \multicolumn{3}{l}{ Primeira diferença } \\
\cline { 2 - 5 } & Defasagens & Estatística t & Defasagens & Estatística t \\
\hline MT & 1 & $-2,432$ & 0 & $-10,016^{* * *}$ \\
MS & 1 & $-2,474$ & 0 & $-10,010^{* * *}$ \\
PR & 1 & $-2,863$ & 0 & $-8,517^{* * *}$ \\
RS & 1 & $-2,809$ & 0 & $-9,149^{* * *}$ \\
GO & 0 & $-2,272$ & 0 & $-12,372^{* * *}$ \\
MG & 1 & $-2,918$ & 0 & $-9,763^{* * *}$ \\
SP & 1 & $-2,934$ & 0 & $-9,504^{* * *}$ \\
SC & 1 & $-3,010$ & 0 & $-9,403^{* * *}$ \\
\hline
\end{tabular}

Fonte: Elaborado pelos autores com base nos resultados da pesquisa.

Nota: $\left.{ }^{* * *}\right)$ denota significância a $1 \%$.

A determinação da especificação do modelo para realização do teste de cointegração levou em consideração a ausência de tendência determinística nos dados e a presença de intercepto apenas na equação de cointegração. Foram incluídas no modelo as séries de preços de todos os estados considerados relevantes neste estudo, ou seja, Mato Grosso, 
Paraná, Rio Grande do Sul, Goiás, Mato Grosso do Sul, Minas Gerais, São Paulo e Santa Catarina (Tabela 2).

Tabela 2 - Teste de Johansen para Identificação do Número de Relações de Cointegração entre as Séries de Preços Analisados

\begin{tabular}{lllll}
\hline Hipótese nula & $\boldsymbol{\tau}_{\text {trace }}$ & $\begin{array}{l}\text { Valor crítico } \\
\text { a 5\% }\end{array}$ & $\boldsymbol{\tau}_{\text {máx }}$ & $\begin{array}{l}\text { Valor crítico } \\
\text { a 5\% }\end{array}$ \\
\hline$r=0$ & 367,260 & 169,599 & 106,730 & 53,187 \\
$r \leq 1$ & 260,529 & 134,678 & 63,286 & 47,078 \\
$r \leq 2$ & 197,242 & 103,847 & 59,562 & 40,956 \\
$r \leq 3$ & 137,680 & 76,972 & 53,535 & 34,805 \\
$r \leq 4$ & 84,1439 & 54,079 & 36,430 & 28,588 \\
$r \leq 5$ & 47,7136 & 35,192 & 24,648 & 22,299 \\
$r \leq 6$ & 23,0655 & 20,261 & 17,261 & 15,892 \\
$r \leq 7$ & 5,80372 & 9,164 & 5,803 & 9,164 \\
\hline
\end{tabular}

Fonte: Elaborado pelos autores com base nos resultados da pesquisa.

Tanto o teste do traço como o do máximo autovalor indicaram a presença de sete vetores de cointegração, já que a hipótese nula não foi rejeitada quando, o que indica que os preços praticados nos mercados analisados seguiram uma tendência comum ao longo do tempo. De modo equivalente à análise gráfica, esses resultados também indicam a participação das regiões analisadas no mercado brasileiro de soja em grão.

No intuito de confirmar a participação das séries de preços como integrantes de um mesmo mercado, foram realizados testes de Razão de Verossimilhança para restrições relacionadas com os parâmetros $\alpha \mathrm{e}$ $\beta$. Todas as restrições impostas aos parâmetros $\beta$ foram rejeitadas a $1 \%$ de significância, o que indica que os oito estados participaram, efetivamente, do equilíbrio de longo prazo no mercado brasileiro de soja em grão, sendo relevantes no espaço de cointegração (Tabela 3). Isto indica que todos os mercados considerados são relevantes no estabelecimento do padrão de equilíbrio de longo prazo, entre os mercados analisados. 
Tabela 3 - Testes de Razão de Verossimilhança de Restrições aos Parâmetros de Cointegração ( $\beta$ 's), Relacionados com cada um dos Estados que Compõem o Espaço de Cointegração

\begin{tabular}{lll}
\hline $\boldsymbol{H}_{\mathbf{0}}: \boldsymbol{\beta}$ ' $\mathbf{=} \mathbf{0}$ & $\boldsymbol{x}^{\mathbf{2}}$ & $\mathbf{P}$-valor \\
\hline $\mathrm{MT}=0$ & 40,586 & 0,000 \\
$\mathrm{MS}=0$ & 41,909 & 0,000 \\
$\mathrm{PR}=0$ & 39,707 & 0,000 \\
$\mathrm{RS}=0$ & 35,910 & 0,000 \\
$\mathrm{GO}=0$ & 47,946 & 0,000 \\
$\mathrm{MG}=0$ & 32,005 & 0,000 \\
$\mathrm{SP}=0$ & 37,604 & 0,000 \\
$\mathrm{SC}=0$ & 40,346 & 0,000 \\
\hline
\end{tabular}

Fonte: Elaborado pelos autores com base nos resultados da pesquisa.

Com relação às restrições impostas sobre os parâmetros , constatase que os coeficientes de ajustamento, referentes aos mercados do Mato Grosso e do Mato Grosso do Sul, foram, conjuntamente, iguais a zero, o que indica exogeneidade fraca e sugere que estes mercados não se ajustam a desequilíbrios transitórios nos demais mercados (Tabela 4).

Tabela 4 - Testes de Razão de Verossimilhança de Restrições aos Parâmetros de Ajustamento ( $\alpha$ 's), Relacionados com cada um dos Estados que Compõem o Espaço de Cointegração

\begin{tabular}{lll}
\hline $\boldsymbol{H}_{\mathbf{0}}: \boldsymbol{\alpha} \mathbf{s} \boldsymbol{\mathbf { s }} \mathbf{0}$ & $\boldsymbol{x}^{\mathbf{2}}$ & $\mathbf{P}$-valor \\
\hline $\mathrm{MT}=0$ & 9,996 & 0,188 \\
$\mathrm{MS}=0$ & 6,337 & 0,500 \\
$\mathrm{PR}=0$ & 31,762 & 0,000 \\
$\mathrm{RS}=0$ & 22,530 & 0,002 \\
$\mathrm{GO}=0$ & 40,778 & 0,000 \\
$\mathrm{MG}=0$ & 33,617 & 0,000 \\
$\mathrm{SP}=0$ & 30,752 & 0,000 \\
$\mathrm{SC}=0$ & 31,125 & 0,000 \\
\hline
\end{tabular}

Fonte: Elaborado pelos autores com base nos resultados da pesquisa. 
Portanto, a análise gráfica do comportamento dos preços, o teste de cointegração e o teste de Razão de Verossimilhança indicaram a existência de integração no mercado brasileiro de soja em grão. A natureza da integração verificada será analisada na seção seguinte, que trata da dinâmica da transmissão de preços.

\subsection{Dinâmica da Transmissão de Preços}

A análise da dinâmica da transmissão de preços entre as regiões produtoras que compõem o mercado brasileiro de soja em grão foi feita a partir das análises das estimativas dos parâmetros do modelo VEC, conforme especificado na equação (7).

Com o objetivo de analisar as relações de longo prazo entre as diferentes regiões que compõem o mercado, procedeu-se à estimação das relações de cointegração, normalizadas em relação ao estado do Mato Grosso. A escolha deste estado deveu-se ao fato de ele ser o principal produtor nacional, o que evidencia sua importância como referência para os preços praticados nos demais estados.

A Tabela 5 mostra as sete relações de equilíbrio de longo prazo, entre os oito estados analisados no período considerado. As relações asseguradas entre as variáveis no equilíbrio de longo prazo foram estabelecidas em termos de pares de mercado e podem ser expressas da seguinte forma: $S C=0,076+0,874 M T ; S P=0,059+0,883 M T$, e assim por diante, para as demais relações estabelecidas. Todos os coeficientes foram significativos a 1\%, e sua interpretação foi obtida, de forma direta, pela observação das relações. Assim, uma variação positiva de $1 \%$ no preço da soja em Mato Grosso levaria ao aumento de 0,874\% em Santa Catarina; 0,883\% em São Paulo; 0,900\% em Minas Gerais, e assim por diante. Vale destacar que esses coeficientes representam as elasticidades de transmissão de preços no longo prazo, já que as séries foram logaritmizadas.

Vale destacar que a magnitude dos coeficientes encontrados está de acordo com o esperado, uma vez que, por refletirem a transmissão de preços entre os mercados, esses coeficientes devem apresentar valores menores ou iguais a 1. Entretanto, em análises aplicadas a outros mercados, há trabalhos em que foram encontrados coeficientes superiores a 1 , fato que se deve à presença de custos de transação elevados. Como exemplo, podem ser citados González-Rivera e Helfand (2001) e Nogueira et al. (2005). Além disso, os coeficientes encontrados não apresentaram grande variabilidade nas magnitudes, o que indica que a transmissão de preço, entre os estados analisados, seguiu um padrão semelhante. 
Tabela 5 - Vetores de Cointegração ( $\beta$ 's) para o Logaritmo das Séries Mensais de Preços de Soja em Grão Recebidos pelos Produtores, no Período de agosto de 1990 a agosto de 2008

\begin{tabular}{llllllll}
\hline & SC & SP & MG & GO & RS & PR & MS \\
\hline \multirow{2}{*}{ MT } & $-0,874$ & $-0,883$ & $-0,900$ & $-0,918$ & $-0,964$ & $-0,920$ & $-1,005$ \\
& $(-10,676)$ & $(-11,197)$ & $(-11,882)$ & $(-18,999)$ & $(-10,790)$ & $(-14,170)$ & $(-11,736)$ \\
& $-0,076$ & $-0,059$ & $-0,074$ & $-0,008$ & $-0,112$ & $-0,083$ & $-0,090$ \\
& $(-1,653)$ & $(-1,342)$ & $(-1,752)$ & $(-0,296)$ & $(-2,226)$ & $(-2,272)$ & $(-1,872)$ \\
\hline
\end{tabular}

Fonte: Elaborado pelos autores com base nos resultados da pesquisa.

Nota: Valores entre parênteses referem-se à estatística t-student (1\%: 2,$57 ; 5 \%$ : 1,96; 10\%: 1,64).

Outro ponto a ser destacado, com relação à magnitude dos coeficientes, é que se espera que os estados mais próximos daquele considerado como importante formador de preços, neste caso Mato Grosso, apresentem maiores parâmetros de cointegração, como de fato observou-se neste estudo, exceção feita ao Rio Grande do Sul e Paraná, que, embora distantes, apresentaram coeficientes elevados em relação aos estados mais próximos, como Minas Gerais, por exemplo.

No intuito de complementar a análise da dinâmica da transmissão de preços, precedeu-se aos testes de Razão de Verossimilhança para as hipóteses de integração perfeita entre os pares de mercados (Tabela 6). Os resultados encontrados mostraram que a hipótese de integração perfeita deve ser rejeitada, já que o valor da estatística do teste foi significativo a $1 \%$, em todos os casos analisados.

Tabela 6 - Testes de Razão de Verossimilhança para a Hipótese de Integração Perfeita entre Pares de Preços

\begin{tabular}{lll}
\hline $\boldsymbol{H}_{\mathbf{0}}$ & $\boldsymbol{x}^{\mathbf{2}}$ & P-valor \\
\hline$\beta_{S C}=\beta_{M T}$ & 44,805 & 0,000 \\
$\beta_{S P}=\beta_{M T}$ & 42,428 & 0,000 \\
$\beta_{M G}=\beta_{M T}$ & 36,924 & 0,000 \\
$\beta_{G O}=\beta_{M T}$ & 26,720 & 0,000 \\
$\beta_{R S}=\beta_{M T}$ & 36,613 & 0,000 \\
$\beta_{P R}=\beta_{M T}$ & 29,419 & 0,000 \\
$\beta_{M S}=\beta_{M T}$ & 52,946 & 0,000 \\
\hline
\end{tabular}

Fonte: Elaborado pelos autores com base nos resultados da pesquisa. 
Segundo González-Rivera e Helfand (2001), a presença de custos de transação faz com que os vetores de cointegração difiram do padrão de integração perfeita, na medida em que captam diferenças regionais em tecnologias, políticas e qualidade do produto. Ainda segundo esses autores, a rejeição da hipótese de integração perfeita resulta das distinções existentes nos custos de transação relacionados com fretes, taxas interestaduais, comissões de vendas, encargos financeiros, dentre outros.

Vale ressaltar que, em outros trabalhos, realizados no intuito de verificar a integração em segmentos distintos da agropecuária brasileira, foram encontrados resultados semelhantes, rejeitando a hipótese de integração perfeita. Entre estes estudos, podem ser citados os de Pereira (2005), que analisou a integração espacial no mercado brasileiro de boi gordo, o de Rosado (2006), cuja análise referiu-se ao mercado de suínos, e o de Nogueira et al. (2005), que investigaram a integração no mercado de café.

As estimativas dos coeficientes $\alpha$ contêm informação relevante para determinar a estrutura espacial do mercado. Conforme pôde ser observado pela significância dos coeficientes de ajustamento estimados, não foi constatada interação entre todos os mercados (Tabela 7). A interpretação desses coeficientes refere-se ao porcentual do desequilíbrio de longo prazo que é corrigido a cada período. Assim, o coeficiente de ajustamento da equação de Santa Catarina, por exemplo, referente a desequilíbrios em seu próprio mercado em relação ao mercado formador de preços, - 0,465, indica que os preços nesse estado respondem, rapidamente, a desequilíbrios internos, já que $46,5 \%$ do desequilíbrio de longo prazo é corrigido a cada período. Os preços praticados na região respondem também a desequilíbrios, ou aos termos de correção de erros, dos estados de Goiás, Rio Grande do Sul e Mato Grosso do Sul, em relação ao mercado formador de preços, embora, nesses casos, o ajuste seja mais lento em razão da magnitude dos coeficientes.

Os estados de São Paulo e Minas Gerais, a exemplo do que se verificou para Santa Catarina, respondem a seus próprios desequilíbrios e a desequilíbrios ocorridos em Goiás, Rio Grande do Sul e Mato Grosso do Sul. Este resultado poderia ser explicado pela importância que os três últimos estados apresentaram para a produção nacional no período analisado (Tabela 8). De fato, Goodwin e Schroeder (1991) encontraram relação negativa entre volume de produção e nível de integração.

O mercado de Goiás, a exemplo dos demais, foi afetado pelos seus próprios desequilíbrios e também por desequilíbrios no Mato Grosso do Sul e em São Paulo, considerando-se um nível de significância de 10\% para o coeficiente de ajustamento desse último. 
O mercado representado pelo estado do Rio Grande do Sul não foi influenciado por desequilíbrios em seu próprio mercado, embora tenha sofrido influência de Goiás e Mato Grosso do Sul.

Os preços praticados no Paraná, a exemplo de estados menos representativos, ajustaram-se a desequilíbrios em Goiás, Mato Grosso do Sul, Rio Grande do Sul, e em seu próprio mercado.

As análises realizadas evidenciam que os preços praticados nos estados de Santa Catarina, São Paulo, Minas Gerais e Paraná apresentaram maior interdependência, uma vez que, se comparados aos de Goiás e Rio Grande do Sul, reagiram ao maior número de desequilíbrios transitórios nas relações de equilíbrio de longo prazo. Esse resultado pode ser explicado pela pequena participação desses estados, exceção feita ao Paraná, na produção nacional durante o período de análise.

Vale destacar que os preços praticados no Mato Grosso do Sul e no Mato Grosso não se ajustaram a desequilíbrios de curto prazo nos demais mercados, o que corrobora os resultados encontrados para os coeficientes $\alpha$ no teste de Razão de Verossimilhança nesses mercados, que indicou sua exogeneidade fraca. Entretanto, optou-se por incluí-los na análise, uma vez que, além de sua importância na produção nacional, o teste de cointegração, que indicou a existência de sete relações de cointegração, e o teste de Razão de Verossimilhança, para os parâmetros $\beta$, indicaram que os oito estados participaram, efetivamente, do equilíbrio de longo prazo, sendo relevantes no espaço de cointegração.

González-Rivera e Helfand (2001) destacaram alguns fatores importantes na determinação da integração de mercados e, portanto, afetariam a dinâmica da transmissão de preços. Segundo os autores, fatores como capital físico e humano, condições de oferta e demanda em cada mercado, políticas públicas e nível de desenvolvimento influenciam a integração de mercados.

Tabela 7 - Coeficientes de Ajustamento do VEC ( $\alpha$ 's), para o Logaritmo das Séries Mensais de Preços de Soja em Grão Recebidos pelos Produtores, no Período de agosto de 1994 a agosto de 2008

\begin{tabular}{ccccccccc}
\hline $\begin{array}{c}\text { Correção } \\
\text { de erros }\end{array}$ & SC & SP & MG & GO & RS & PR & MS & MT \\
\hline SC-MT & $\mathbf{- 0 , 4 6 5}$ & $-0,004$ & $-0,106$ & 0,080 & $-0,089$ & 0,054 & 0,038 & $-0,052$ \\
& $(-3,953)$ & $(-0,035)$ & $(-0,906)$ & $(0,737)$ & $(-0,760)$ & $(0,560)$ & $(0,330)$ & $(-0,508)$ \\
\hline SP-MT & 0,004 & $\mathbf{- 0 , 4 1 7}$ & 0,038 & $\mathbf{0 , 2 8 4}$ & 0,160 & 0,055 & 0,036 & $-0,061$ \\
& $(0,025)$ & $(-2,428)$ & $(0,227)$ & $(1,815)$ & $(0,951)$ & $(0,402)$ & $(0,221)$ & $(-0,415)$ \\
\hline MG-MT & $-0,086$ & 0,111 & $\mathbf{- 0 , 3 2 3}$ & 0,074 & 0,042 & 0,075 & 0,024 & 0,060 \\
\hline
\end{tabular}


(continuação)

\begin{tabular}{ccccccccc}
\hline & $(-0,995)$ & $(1,257)$ & $(-3,724)$ & $(0,915)$ & $(0,484)$ & $(1,061)$ & $(0,285)$ & $(0,787)$ \\
\hline \multirow{2}{*}{ GO-MT } & $\mathbf{- 0 , 3 7 4}$ & $\mathbf{- 0 , 4 7 4}$ & $\mathbf{- 0 , 3 0 0}$ & $\mathbf{- 0 , 7 7 4}$ & $\mathbf{- 0 , 4 1 2}$ & $\mathbf{- 0 , 3 9 5}$ & $-0,183$ & $-0,011$ \\
& $(-2,981)$ & $(-3,588)$ & $(-2,318)$ & $(-6,418)$ & $(-3,186)$ & $(-3,705)$ & $(-1,448)$ & $(-0,098)$ \\
\hline \multirow{2}{*}{ RS-MT } & $\mathbf{0 , 2 4 8}$ & $\mathbf{0 , 2 8 6}$ & $\mathbf{0 , 2 6 2}$ & $-0,088$ & $-0,197$ & $\mathbf{0 , 2 1 6}$ & 0,106 & 0,056 \\
& $(2,003)$ & $(2,260)$ & $(2,112)$ & $(-0,764)$ & $(-1,587)$ & $(2,114)$ & $(0,872)$ & $(0,518)$ \\
\hline \multirow{2}{*}{ PR-MT } & 0,041 & $-0,079$ & $-0,284$ & $-0,281$ & $-0,080$ & $\mathbf{- 0 , 5 6 6}$ & $-0,210$ & 0,114 \\
& $(0,214)$ & $(-0,402)$ & $(-1,465)$ & $(-1,557)$ & $(-0,412)$ & $(-3,546)$ & $(-1,108)$ & $(0,667)$ \\
\hline \multirow{2}{*}{ MS-MT } & $\mathbf{0 , 3 6 6}$ & $\mathbf{0 , 3 0 1}$ & $\mathbf{0 , 4 3 1}$ & $\mathbf{0 , 3 4 3}$ & $\mathbf{0 , 2 9 6}$ & $\mathbf{0 , 2 6 5}$ & $-0,041$ & 0,094 \\
& $(4,233)$ & $(3,406)$ & $(4,982)$ & $(4,245)$ & $(3,425)$ & $(3,723)$ & $(-0,488)$ & $(1,237)$ \\
\hline
\end{tabular}

Fonte: Elaborado pelos autores com base nos resultados da pesquisa.

Nota: Valores entre parênteses referem-se à estatística t-student (1\%: 2,57; 5\%: 1,96; 10\%: 1,64).

Neste sentido, buscaram-se informações sobre variáveis que representem alguns desses aspectos nos mercados (estados) incluídos neste estudo, no intuito de esclarecer os resultados encontrados para o padrão da dinâmica da transmissão de preços. Essas e outras variáveis têm sido incluídas em estudos de análise de integração de mercados, no sentido de ampliar a discussão e elucidar os resultados encontrados.

A densidade de rodovias pavimentadas, ${ }^{2}$ proxy para capital físico, é um importante fator na determinação da dinâmica da transmissão de preços, uma vez que tem efeito sobre os custos de transação. De fato, os estados que apresentaram maior interdependência - Santa Catarina, São Paulo, Minas Gerais e Paraná - foram os que apresentaram densidade de rodovias mais elevada (Tabela 8 ).

Tabela 8 - Determinantes da dinâmica da transmissão de preços de soja em grão recebidos pelos produtores, no período de agosto de 1994 a agosto de 2008

\begin{tabular}{llllll}
\hline Estados & $\begin{array}{l}\text { Densidade } \\
\text { rodovias } \\
(\mathbf{1 9 9 6 - 2 0 0 4 )}\end{array}$ & $\begin{array}{l}\text { Parcela do } \\
\text { PIB agro- } \\
\text { pecuário } \\
\mathbf{( 1 9 9 5 - 2 0 0 7}\end{array}$ & $\begin{array}{l}\text { PIB per capita } \\
\mathbf{( 1 9 9 5 - 2 0 0 7 )} \\
\mathbf{\text { Rm }} \mathbf{2 0 0 0}\end{array}$ & $\begin{array}{l}\text { Produtividade } \\
\text { média (1995- } \\
\mathbf{2 0 0 7 )} \mathbf{~ e m ~} \mathbf{~ k g} \\
\text { por ha }\end{array}$ & $\begin{array}{l}\text { Produção } \\
\text { média } \\
\text { (1995- } \\
\mathbf{2 0 0 8 )}\end{array}$ \\
\hline MG & 0,01 & 22,59 & $6.232,38$ & 2.823 & $11,150,7$ \\
MS & 0,01 & 21,93 & $5,956,74$ & 2,403 & $3,267,6$ \\
PR & 0,09 & 10,69 & $7.363,28$ & 2.681 & $8.763,1$ \\
RS & 0,04 & 10,77 & $8,444,88$ & 1.817 & $6.281,3$ \\
GO & 0,03 & 14,32 & $4.964,93$ & 2.525 & $4.698,6$ \\
\hline
\end{tabular}

2 Obtida ao dividir a média da extensão em $\mathrm{km}$ de estradas municipais, estaduais e federais pavimentadas para o período 1996-2004 pela área total $\mathrm{em} \mathrm{km}^{2}$ de cada estado. 
(continuação)

\begin{tabular}{llllll}
\hline MG & 0,04 & 8,22 & $6.043,63$ & 2.378 & $1.897,5$ \\
SP & 0,11 & 3,34 & $10.687,95$ & 2.414 & $1.443,6$ \\
SC & 0,07 & 10,34 & $8.202,31$ & 2.361 & 642,1 \\
\hline
\end{tabular}

Fonte: Elaborado pelos autores com base nos resultados da pesquisa.

Notas: Os dados para o cálculo da densidade das rodovias foram obtidos do Anuário Estatístico dos Transportes Terrestres, elaborado pela Empresa Brasileira de Planejamento dos Transportes - GEIPOT (2010), para o período 1996-2000, e pela Agência Nacional dos Transportes Terrestres para o período 2001-2004. Os dados para o cálculo da participação do PIB agropecuário e do PIB per capita foram obtidos no Instituto de Pesquisa Econômica Aplicada (IPEA). Os dados de produção e produtividade média foram obtidos na Companhia Nacional de Abastecimento (Conab) e no Instituto Brasileiro de Geografia e Estatística (IBGE), respectivamente.

A produtividade média por hectare atuou como proxy para capital humano devido à relação positiva entre essas duas variáveis (GONZÁLEZ-RIVERA; HELFAND, 2001). Entretanto, todos os estados apresentaram produtividades médias semelhantes, o que não permitiu relacionar produtividades elevadas a maior nível de interdependência.

A participação do Produto Interno Bruto (PIB) agropecuário no PIB total e o PIB per capita provêm maneiras alternativas de medir o nível de desenvolvimento em cada estado que, segundo González-Rivera e Helfand (2001), capturam diferenças na quantidade e qualidade da infraestrutura de comercialização em cada estado que poderiam reduzir custos de transação e elevar a integração. Neste caso, níveis elevados do PIB per capita e baixas participações do PIB agropecuário estariam associados à maior integração.

Os estados destacados como aqueles que apresentaram maior nível de interdependência foram os que tiveram menor participação do PIB agropecuário. Quando observam-se os níveis de renda per capita, esses estados estão entre os que apresentaram níveis de renda mais elevados, exceção feita a Minas Gerais, que apresentou renda per capita inferior à dos estados como Mato Grosso e Rio Grande do Sul, considerados menos interdependentes pelos resultados encontrados para os coeficientes de ajustamento.

Portanto, os dados levantados sobre a disponibilidade de capital físico e os níveis de desenvolvimento indicam que esses fatores, possivelmente, possuem relação com o padrão de interdependência verificado entre os preços. Esses indícios mostram a importância de investimentos em infraestrutura de comercialização como forma de promover maior integração dos mercados agropecuários. 


\section{Considerações Finais}

A análise de integração realizada neste trabalho permitiu concluir que os mercados analisados estão integrados entre si, ainda que de forma imperfeita, e que há relação de equilíbrio, no longo prazo, entre os preços recebidos pelos produtores nessas regióes. Pode-se dizer que existe um fluxo comum e único de informações entre os agentes participantes desse mercado.

Como não houve integração perfeita, rejeitou-se a validade da lei do preço único. Esse fato pode ser atribuído à existência de custos de transação e à diferenciação desses custos nos vários mercados analisados.

Do mesmo modo, não foi verificada interdependência elevada dos preços praticados, visto que nenhum dos mercados considerados apresentou ajustes a desequilíbrios de longo prazo em todos os demais. Deve-se destacar, entretanto, que os estados de Santa Catarina, São Paulo, Minas Gerais e Paraná apresentaram maior interdependência, já que foram os mais suscetíveis a desequilíbrios ocorridos nos demais.

A ausência de integração perfeita indica que choques de oferta e demanda que surgiram nos mercados analisados não foram integralmente transmitidos aos demais. Adicionalmente, o padrão verificado para a dinâmica da transmissão de preços indicou que nenhum dos mercados analisados foi suscetível às condições de oferta e demanda em todos os outros.

Algumas características de cada mercado podem ser apontadas como responsáveis pela ausência de integração perfeita e interdependência elevada. As disponibilidades de capital físico e a quantidade e qualidade da infraestrutura de comercialização, medidas pela participação do PIB agropecuário no PIB total e pelo PIB per capita, apresentaram grande variabilidade entre os estados, o que evidencia que eles estão sujeitos a custos de transação significativamente distintos. Ademais, os estados que apresentaram maior interdependência de preços, de acordo com os resultados, foram os que apresentaram melhores condições de infraestrutura e melhores níveis de desenvolvimento, o que indica a importância desses elementos como prováveis determinantes da integração de mercados.

O conhecimento a respeito da integração de mercados contribui para melhor planejamento da comercialização da safra por parte dos produtores. E de interesse direto dos agentes ligados à produção e à comercialização de soja saber como os preços praticados em seu estado são influenciados pelos preços nos demais. Esse conhecimento permite aos agentes, por exemplo, prever como os preços praticados em seu estado reagem a uma supersafra ou a uma quebra de safra nas principais 
regiões produtoras do País, fornecendo subsídios para que os agentes tomem decisões de comercialização e produção.

Por fim, deve-se destacar que a ausência de elevada interdependência dos mercados revela a necessidade de estratégias e políticas que atuem na redução dos custos de transação como forma de promover o processo de integração. Nesse sentido, investimentos em infraestrutura de comercialização e uma política fiscal uniforme entre os estados seriam elementos importantes. Esses esforços, ao favorecerem o processo de integração, elevariam a eficiência no mercado brasileiro de soja em grão.

\section{Referências}

AGÊNCIA NACIONAL DOS TRANSPORTES TERRESTRES - ANTT. Disponível em: $<$ http://www.antt.gov.br/aett/aett_2005/FSCOMMAND/Estatisticas/1.1.1.htm>. Acesso em: 15 fev. 2010.

ANUÁRIO DA AGRICULTURA BRASILEIRA - AGRIANUAL. Soja: balanço mundial. São Paulo: FNP Consultoria E Comércio, 2010.

BRASIL. Ministério do Desenvolvimento, Indústria e Comércio Exterior. Alice Web, 2009. Disponível em: <http://www.midic.gov.br. Acesso em 14 jan. 2010>.

COELHO, A. B. A cultura do algodão e a questão da integração entre preços internos e externos. Revista de Economia e Sociologia Rural, v. 42, n. 1, p. 153-169, 2004.

COMPANHIA NACIONAL DE ABASTECIMENTO - CONAB. Central de informações agropecuárias: safras. Disponível em:<http://www.conab.gov.br>. Acesso em: 15 nov. 2009.

CUNHA, D. A. Integração de preços no mercado internacional de café, p. 105, 2008. Dissertação (Mestrado em Economia Aplicada) - Universidade Federal de Viçosa, Viçosa, MG, 2008.

DE ZEN, S. Integração entre os mercados de boi gordo e de carne bovina nas regiões CentroOeste e Sudeste do Brasil, p. 81, 1997. Dissertação (Mestrado em Economia Aplicada) - Escola Superior de Agricultura Luiz de Queiroz, Piracicaba, SP, 1997.

DICKEY, D. A.; FULLER, W. A. Likelihood ratio statistics for autoregressive time series with a unit root. Econometrica, v. 49, n. 4, p. 1057-1072, 1981.

EMPRESA BRASILEIRA DE PLANEJAMENTO DOS TRANSPORTES - GEIPOT. Anuário estatístico dos transportes terrestres. Disponível em: <http://www.geipot.gov.br/anuario2001/index.htm >. Acesso em: 16 jan. 2010.

ENDERS, W. Applied econometric time series. New York: John Wiley, 1995.

FACKLER, P. L.; GOODWIN, B. K. Spatial price analysis. In: RAUSSER, G. C.; GARDEN, B. L. (Ed.). Handbook of agricultural economics. Amsterdam: North-Holland Press, p. 971-1024, 2001.

FAMINOW, M. D.; BENSON, B. L. Integration of spatial markets. American Journal of Agricultural Economics, v. 72, n. 1, p. 49-62, 1990.

FUNDAÇÃO GETÚLIO VARGAS - FGV. FGVDADOS: preços agropecuários. Disponível em: <http://fgvdados.fgv.br. Acesso em 20 out. 2008>. 
GONZÁLEZ-RIVERA, G.; HELFAND, S. M. Economic development and the determinants of spatial integration in agricultural markets, Working Paper 28, Riverside: University of California, 2001.

GOODWIN, B. K.; SCHROEDER, T. C. Cointegration tests and spacial price linkages in regional cattle markets. American Journal of Agricultural Economics, v. 73, n. 2, p. 452-464, 1991.

INSTITUTO BRASILEIRO DE GEOGRAFIA E ESTATÍSTICA - IBGE. Banco de dados agregados - SIDRA. Disponível em: < http://www.sidra.ibge.gov.br/>. Acesso em: 22 fev. 2010.

INSTITUTO DE PESQUISA ECONÔMICA APLICADA - IPEA. Base de dados regionais. Disponível em: <http://www.ipeadata.gov.br>. Acesso em: 16 jan. 2010.

JOHANSEN, S. Statistical analysis of cointegration vectors. Journal of Economic Dynamics and Control, v. 12, n. 2-3, p. 231-254, 1988.

KRUGMAN, P. R.; OBSTFELD, M. Economia internacional: teoria e política. São Paulo: Pearson Addison Wesley, 2005.

MARGARIDO, M. A.; TUROLLA, F. A.; FERNANDES, J. M. Análise da elasticidade de transmissão de preços no mercado internacional de soja. Pesquisa 8 Debate, v. 12 , n. 2, p. 5-40, 2001.

MATTOS, L. B. Efeitos de custos de transação sobre a integração espacial de mercados regionais de carne de frango no Brasil. 2008. 162 f. Tese (Doutorado em Economia Aplicada) - Universidade Federal de Viçosa, Viçosa, MG, 2008.

NOGUEIRA, F. T. P.; AGUIAR, D. R. D.; LIMA, J. E. Integração espacial no mercado brasileiro de café arábica. Nova Economia, v. 15, n. 2, p. 91-112, 2005.

PEREIRA, L. R. R. Integração espacial no mercado brasileiro de boi gordo. 2005. p. 166. Tese (Doutorado em Economia Aplicada) - Universidade Federal de Viçosa, Viçosa, MG, 2005.

ROSADO, P. L. Integração espacial entre os mercados brasileiros de suínos, p. 117, 2006. Tese (Doutorado em Economia Aplicada) - Universidade Federal de Viçosa, Viçosa, MG, 2006.

SANTANA, A. C. Comercialização e integração de mercado na pecuária de corte do Estado do Pará. In: CONGRESSO BRASILEIRO DE ECONOMIA E SOCIOLOGIA RURAL, 36., 1998, Poços de Caldas. Anais... Brasília: SOBER, 1998.

Recebido: $13 / 10 / 2009$.

Aceito: 10/08/2010. 